\title{
A violence epidemic model to study trend of domestic violence, a study of tamale metropolis
}

\author{
Charles Sebil $^{1 *}$, Dominic Otoo ${ }^{2}$ \\ ${ }^{l}$ Mathematics department, Kwame Nkrumah university of science and technology, Kumasi, Ghana \\ ${ }^{2}$ Mathematics department, university of energy and natural resources, Sunyani, Ghana \\ *Corresponding author E-mail: dominicotoo@rocketmail.com
}

Copyright $($ C) 2014 Charles Sebil, Dominic Otoo. This is an open access article distributed under the Creative Commons Attribution License, which permits unrestricted use, distribution, and reproduction in any medium, provided the original work is properly cited.

\begin{abstract}
There is a growing concern about the rise of violence on the streets and the media around the world, the possibility of an individual to be affected by violence at home is an undeniable reality facing most families around the globe. Domestic violence can take many forms including physical, psychological, sexual, and economic. It not only has devastating physical and psychological consequences on its victims, but can seriously damage the foundations of the family leading to its disintegration. There is therefore the need to find out the trend of spread in our communities, since it has the potential to slow down productivity in any society. The study used a simple continuous model for the spread of Domestic Violence, using Ordinary Differential Equations. A mathematical model is inspired from the spread of Domestic Violence in Tamale Metropolis in which the interaction of the widespread is likely to be minimized. A modeling technique of Abusive, Susceptible and Violence Victims (ASV), similar to the Susceptible, Infectious and Recovered (SIR) model in Epidemics, is used for formulating the spread of Domestic Violence as a system of Differential Equations. The system of Differential Equations is analyzed by linearization of nonlinear systems and nondimensionlization to predict the behaviour of the spread of Domestic Violence.
\end{abstract}

Keywords: Abusive, domestic violence, epidemic model, infectious and recovered susceptible and violence victims.

\section{Introduction}

Tamale, one of the ten regional capitals of Ghana is a bustling Regional Capital of the Northern Region about 400 miles north of the Atlantic Coast in West Africa. The geographical area of Tamale is about 750 kilometer square (289.58 square miles). The economy of the area is predominantly Agricultural. The region has a population of Two million four hundred and sixty-eight thousand, five hundred and fifty seven $(2,468,557)$ out of which One million two hundred and fifty seven thousand eight hundred and fifty-five $(1,257,855)$ are females and the rest males. The study area 'Tamale' constitutes about $42 \%$ of the region's population. Most people in capital are Muslims and they practice the polygamous system of marriage with large family size. The dominant tribe is the Dagombas but there are other tribes like Gonja, Mamaprusi, Konkomba, Hausa, Dagarti, and Grusi. Globally, in 90 percent of the cases of Domestic Violence, the victim is a woman and 90 percent of the time the abuser is a man. Violence against women and children continues to be a global epidemic that kills, tortures, and maims - physically, psychologically, sexually and economically. It is one of the most pervasive of human rights violations, denying women and children equality, security, dignity, self-worth, and their right to enjoy fundamental freedoms. Violence against women and children is present in every country, cutting across boundaries of culture, class, education, income, ethnicity and age. Domestic violence has been outlined by Amnesty International as most violent attacks on an individual or group of people or women. It involves physical, sexual and psychological violence in the family including battering, sexual abuse of female children in the household, dowry related violence, marital rape, female genital mutilation and other traditional practices harmful to any member of the household. Domestic violence may be exhibited by any member of the household. 


\section{Related works}

As defined by [1], "Violence is an act carried out with the intention of perceived intention of physically hurting another person". It can also be defined as the exercise of power in order to impose one's will on a person or to have ones will with a thing. Also Seager [9], defined "domestic violence is a leading cause of injury and death to women and children and worldwide. It is often ignored or even condoned by the state on the grounds that they are 'private' matter". She further explores domestic violence as a tool of power used to sustain particular sexual, family and household structures and to keep women subordinate to them.

Montroll, [7] makes the connection between physical and population trajectories clear by proposing laws of social dynamics based on Newton's laws of mechanics. Epidemic models have been used by many researchers to estimate the spread of diseases.

Grais [2] used epidemic model to estimate the transmission of measles in Niamey, Niger. Spatial and spatiotemporal epidemiology has been used to model the influenza dynamics in the North America [3].

Li [5] used SIR epidemic model to test its stability and bifurcation, [10] has also considered some properties of simple epidemic model of SIR, and the global stability analysis with discretization approach for an age-structured multi-group using SIR model [4].

[6] used log-linear model on Capture-Recapture method to estimate the spread of measles, [8] modeled attack and outbreak of Zombies and had comparable results.

\section{Scope of the study}

The study area, Tamale is the regional capital of the northern region the region with the largest land area and the third most populated region after greater Accra and Ashanti regions respectively. The region has a population of Two million four hundred and sixty-eight thousand, five hundred and fifty seven $(2,468,557)$ out of which One million two hundred and fifty seven thousand eight hundred and fifty-five $(1,257,855)$ are females and the rest males. The study area 'Tamale' constitutes about $42 \%$ of the region's population. Data for the study was obtained from office of Domestic Violence and Victims Support Unit (DOVVSU) in Tamale covering the reported annual incidence from 1999-2011.

\section{Mathematical formulation of violence epidemic model (ASV)}

The ASV model just as the SIR Model in epidemiology will be used for the analysis. The victims will be mainly restricted to Tamale Metropolis. Therefore, we assume that the committing of Domestic Violence occurs mostly within a high-risk population group, such as men, women and children. Besides, Domestic Violence Victims, who are at the end-stage of the Domestic Violence infection, are strictly isolated from Abusive and susceptible population; therefore, they no longer cause any form of Violence. To simplify a real problem, we assume;

\section{Assumptions:}

1) A particular population, which is reasonably restricted, is at high-risk to Domestic Violence by contact only.

2) The population is uniformly mixed, so the probability of a person been a victim of Domestic Violence equally exists to every single individual within the community.

3) Once victims (Women and Children) are classified into Domestic Violence Victims, they are no longer engaged in the spread of the violence.

4) There are no subtractions of the population except for Violence-induced death.

For the Domestic Violence victim modeling, we will consider a group of susceptible individuals; therefore a number of susceptible individuals (Women and Children) are introduced into a larger susceptible population. Besides, the infected individuals develop themselves into a group of Domestic Violence Victims, the end-stage of the violence. The population of each group will change respect to time while the violence progresses. Therefore, we will think of the change of the population as a mass balance idea,

Rate of change in Domestic Violence population = population growth - population loss.

Then, the terms for the population growth and population loss can be defined by the interaction of each population group in the transmission of violence. To mathematically translate, we will introduce a system of ordinary differential equations. Let's declare variables before creating ODEs.

Definition of Variables and Parameters

- $\quad N$ - Total population of a community.

- $\quad \mu$ - Recruitment rate to a population.

- $\quad \beta$-Conversion rate (from Susceptible to Victim).

- $S(t)$ - Number of Susceptible individuals/unreported domestic violence victims at time $(t)$.

- $V(t)$ - Number of Reported Domestic Violence Victim's at time $(t)$.

- $A(t)$ - Number of Abusive individuals at time $(t)$. 
- $\quad \alpha$-Susceptible/Unreported Victims rate.

- $\delta$ - Violence-induced death rate.

The number of each population changes in time, so it can be expressed as a function of time, and the total population, $N(t)$, consists of three sub-classes, $A(t), S(t)$ and $V(t)$. First, let's consider the rate of change in the susceptible individuals $S(t)$, which represents all women and children in the population who are exposed to Domestic Violence. Since there is a population growth at a rate, $\mu$, new incomers will immediately belong to the Abusive group, $A(t)$. then, these individuals may be Susceptible by violent contact with in the Abusive group. Note that the infection occurs at a rate proportional to the number of the Susceptible and Abusive; that is $\alpha A S$, where a universal parameter, $\alpha$ represents all infective factors according to our assumptions. Thus, once individuals are infected, there will be the subtraction of population at the rate, $\alpha A S$, from $A(t)$. Along with the population growth at the rate, $\mu$, we finally have the equation $\frac{d A}{d t}=\mu-\alpha A(t) S(t)$. Simultaneously, the infection will also invoke addition to $S(t)$ at the same rate, $\alpha A S$. In the same manner, the development of Susceptible individuals, $S(t)$, into Domestic Violence Victim at a certain rate will result in the subtraction of population from $S(t)$ and the addition of population to the Domestic Violence Victim, $V(t)$. Let $\beta$ be the conversion rate from the Susceptible to Domestic Violence Victim, then the subtraction and the addition from $S(t)$ to $V(t)$ can be expressed as $\beta S$. Finally, Domestic Violence Victim may die at a rate $\delta$, which is the violence-induced death rate. As a result, a simple model can be formed as a system of deferential equations.

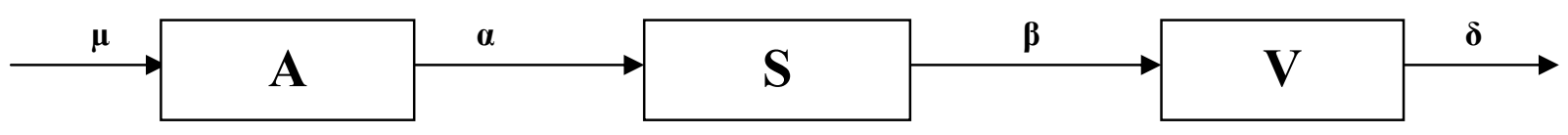

Fig. 1: A simple Domestic Violence Model

$\frac{d A}{d t}=\mu-\alpha A(t) S(t)$

$\frac{d S}{d t}=\alpha A(t) S(t)-\beta S(t)$

$\frac{d V}{d t}=\beta S(t)-\delta V(t)$

We will note that the system of the differential equations is a nonlinear 3-dimensional system. Also, we will note that equation (3.10) is an inhomogeneous equation since it has a recruitment rate $\mu$ per unit time to the Abusive class. As a result, the total population $N(t)=A(t)+S(t)+V(t)$

The $\mu$ term is much like a "forcing term" which often arises from an external force in a physical application.

\subsection{Analysis using the violence epidemic (ASV) model}

Unfortunately, we cannot explicitly solve the system of the differential equations, (3.1), (3.2) and (3.3). Instead, we can import an analytic approach to study the behavior of the infection. In the system, (3.1), (3.2) and (3.3), $\frac{d A}{d t}$ and $\frac{d S}{d t}$ are independent on Domestic Violence Victims, $V$. In other words, Domestic Violence Victims no longer affect the primary infection, so we will consider $\frac{d A}{d t}$ and $\frac{d S}{d t}$ only. Then, we have an equivalent 2-dimensional system for our model,

$\frac{d A}{d t}=\mu-\alpha A S$

$\frac{d S}{d t}=\alpha A S-\beta S$

From $\frac{d A}{d t}=\mu-\alpha A S$, since $\frac{d A}{d t}$

$0=\mu-\alpha A S$

$\alpha A S=\mu$

$A=\frac{\mu}{\alpha S}$

Also from $\frac{d S}{d t}=\alpha A S-\beta S$, since $\frac{d S}{d t}=0$ 
$0=\alpha A S-\beta S$

$\beta S=\alpha A S$

$A^{*}=\frac{\beta}{\alpha}$

Therefore, from equation (4.03)

$S^{*}=\frac{\mu}{\beta}$

Primarily, we're interested in equilibrium, if any, at which both $A$ and $S$ do not change; that is, neither increasing nor decreasing. For that, we need to find $A^{*}$ and $S^{*}$ such that both $\frac{d A}{d t}=0$ and $\frac{d S}{d t}=0$. To do that, we will look at the intersection of $A$ and $S$

$S$

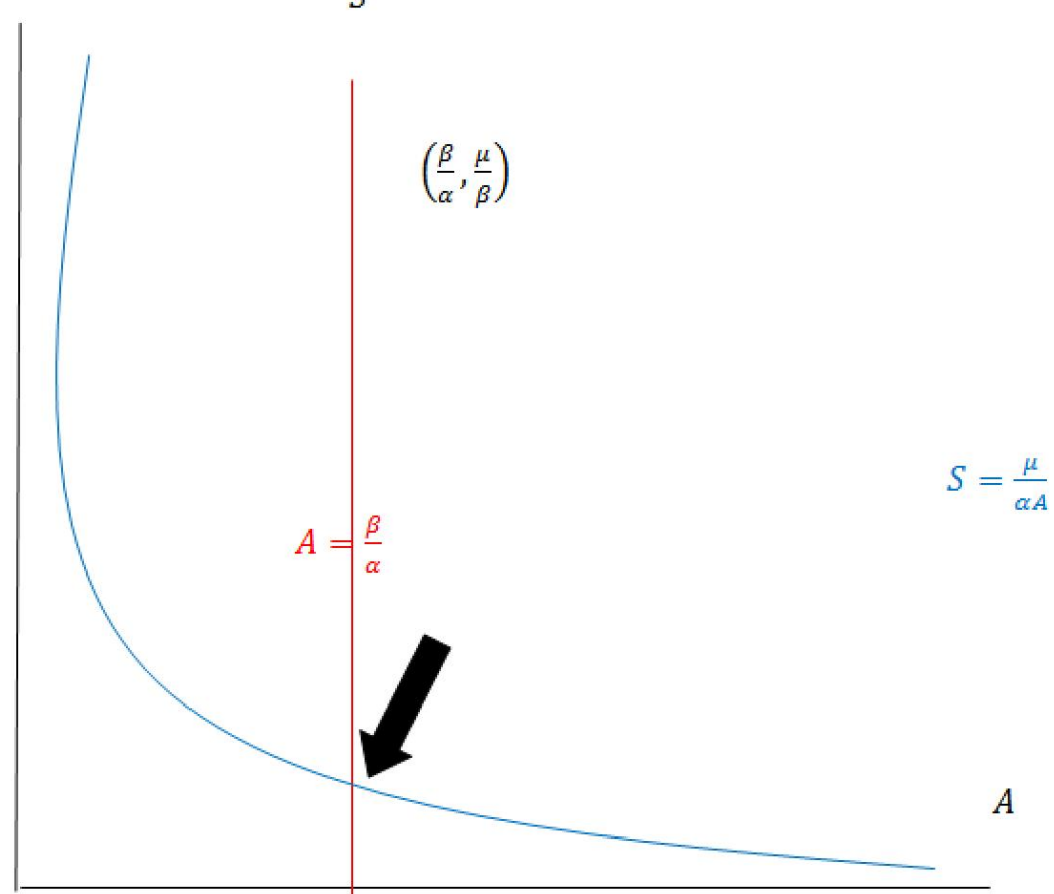

Fig. 2: A graph of intersection of A and S

We have one equilibrium point $E\left(A^{*}, S^{*}\right)=\left(\frac{\beta}{\alpha}, \frac{\mu}{\beta}\right)$ in the first quadrant. Thus, when $A^{*}=\frac{\beta}{\alpha}$ and $S^{*}=\frac{\mu}{\beta}$, the infection results a condition in which there is no change in the population of the Abusive individuals and the Susceptible and unreported victims of domestic violence. However, this is not quite enough information to predict the behavior of the violence. We need to know whether the equilibrium is stable or unstable. Since the system is nonlinear, we need to linearize the system at the equilibrium $E\left(A^{*}, S^{*}\right)$ to determine its stability by taking the Jacobian matrix, which is defined as, $J\left(A^{*}, S^{*}\right)=\left(\begin{array}{ll}\frac{\partial f}{d A} & \frac{\partial f}{d S} \\ \frac{\partial g}{\partial A} & \frac{\partial g}{\partial S}\end{array}\right)_{\mid\left(A^{*}, S^{*}\right)}$

Where, $\frac{d A}{d t}=f(A, S)$ and $\frac{d S}{d t}=g(A, S)$

Then, the linearization of the system at the equilibrium $E$ can be calculated as,

$\frac{\partial f}{\partial A}=-\alpha S$ and $\frac{\partial f}{\partial S}=-\alpha A$

$\frac{\partial g}{\partial A}=\alpha S$ and $\frac{\partial g}{\partial S}=\alpha A-\beta$ 


$$
\begin{gathered}
J(A, S)=\left(\begin{array}{cc}
-\alpha S & -\alpha A \\
\alpha S & \alpha S-\beta
\end{array}\right) \\
J\left(\frac{\beta}{\alpha}, \frac{\mu}{\beta}\right)=\left(\begin{array}{cc}
-\frac{\alpha \mu}{\beta} & -\beta \\
\frac{\alpha \mu}{\beta} & 0
\end{array}\right) \\
|A-\lambda I|=0 \\
\left|\left(\begin{array}{cc}
-\frac{\alpha \mu}{\beta} & -\beta \\
\frac{\alpha \mu}{\beta} & 0
\end{array}\right)-\lambda\left(\begin{array}{ll}
1 & 0 \\
0 & 1
\end{array}\right)\right|=0 \\
\left|\begin{array}{cc}
-\frac{\alpha \mu}{\beta}-\lambda & -\beta \\
\frac{\alpha \mu}{\beta} & -\lambda
\end{array}\right|=0 \\
-\lambda\left(-\frac{\alpha \mu}{\beta}-\lambda\right)-(-\beta)\left(\frac{\alpha \mu}{\beta}\right)=0 \\
\lambda^{2}+\left(\frac{\alpha \mu}{\beta}\right) \lambda+\alpha \mu=0
\end{gathered}
$$

From the general Quadratic formula, $\lambda=\frac{-b \pm \sqrt{b^{2}-4 a c}}{2}$

Where $a=1, b=\frac{\alpha \mu}{\beta}$ and $c=\alpha \mu$

$\lambda=\frac{-\frac{\alpha \mu}{\beta} \pm \sqrt{\left(\frac{\alpha \mu}{\beta}\right)^{2}-4 \alpha \mu}}{2}$

By looking at the eigenvalues of this matrix, we can determine the stability of the original nonlinear system at the equilibrium. This implies the original nonlinear system would behave like the linearized system at the equilibrium. The eigenvalues of the Jacobian, $\lambda$ calculated as, $\lambda=\frac{-\frac{\alpha \mu}{\beta} \pm \sqrt{\left(\frac{\alpha \mu}{\beta}\right)^{2}-4 \alpha \mu}}{2}$

Since the eigenvalues, $\lambda$ are determined by the parameters, we need to break down into two cases to determine the stability of the equilibrium.

First, when $\frac{\alpha \mu}{\beta^{2}} \geq 4$

The eigenvalues $\lambda$ have two negative real parts since we know, $\frac{\alpha \mu}{\beta}>\sqrt{\left(\frac{\alpha \mu}{\beta}\right)^{2}-4 \alpha \mu}>0$

Hence the system forms a nodal sink at the equilibrium. That implies the system have the behavior of exponential decay at the equilibrium, $E\left(A^{*}, S^{*}\right)=\left(\frac{\beta}{\alpha}, \frac{\mu}{\beta}\right)$, which is asymptotically stable. Thus, every solution of the system will approach the stable equilibrium point, $A^{*}=\frac{\beta}{\alpha}$ and $S^{*}=\frac{\mu}{\beta}$, as time $t$ goes to infinity.

Secondly when $\frac{\alpha \mu}{\beta^{2}}<4$

The eigenvalues, $\lambda$, have complex parts along with a negative real part, hence the system forms a spiral sink at the equilibrium. That means the system have a certain form of oscillation behavior at equilibrium, $E\left(A^{*}, S^{*}\right)=\left(\frac{\beta}{\alpha}, \frac{\mu}{\beta}\right)$ while its solutions still move to stable direction. In other words, the system behaves in a damped oscillatory manner with a certain period determined by the parameters. Given the model parameters, the period of the oscillation plays a role for us to predict the further behavior of the infection. 

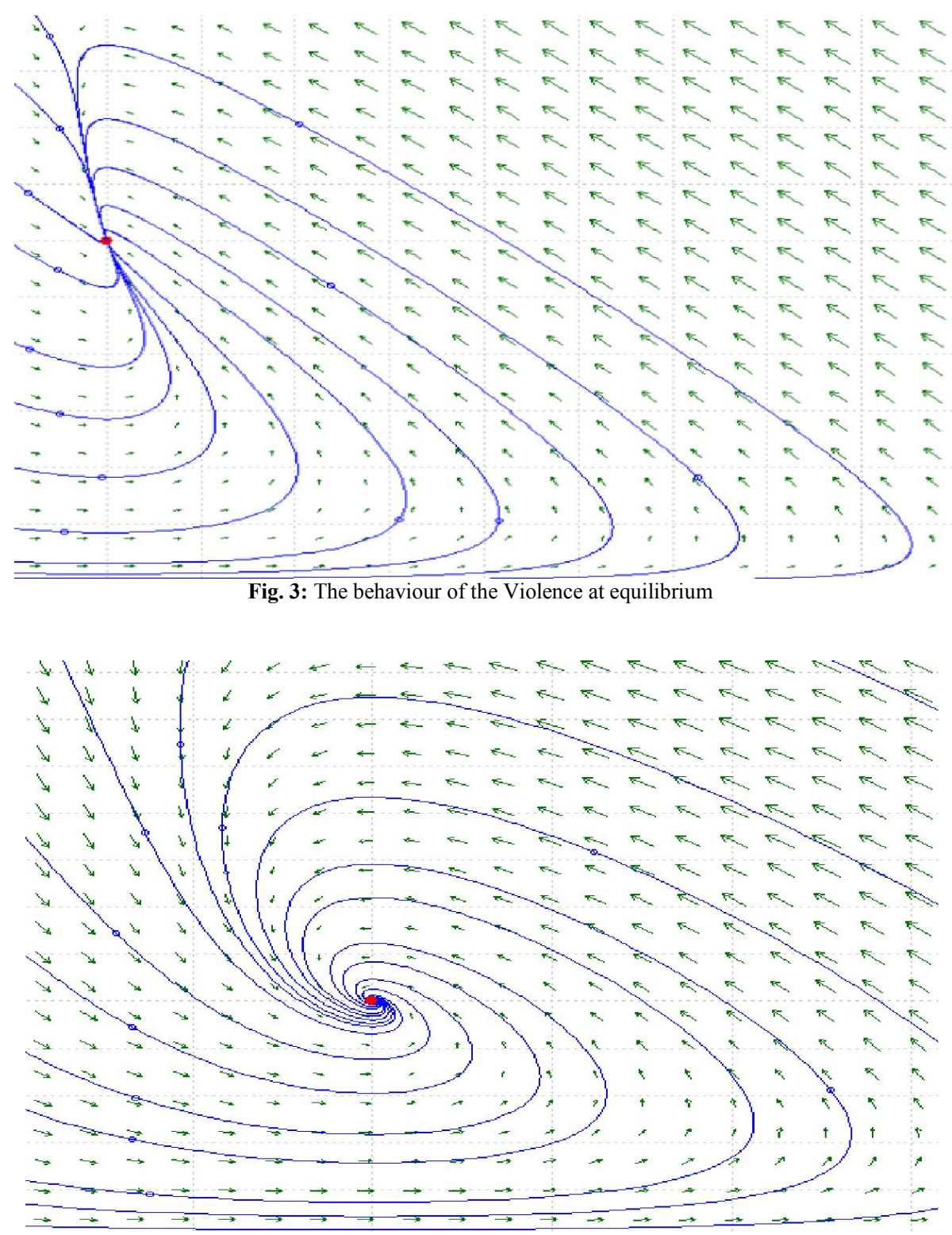

Fig. 4: The behavior of the Violence at equilibrium

Interestingly, in either case, we can observe a stable equilibrium point, $A^{*}=\frac{\beta}{\alpha}$ and $S^{*}=\frac{\mu}{\beta}$, where the violence would have a steady state. Now, an interesting question comes out. The parametric conditions with inequality, (4.7) and (4.8), come purely from the mathematical analysis and we have no clear insight about how they are related to the behaviour of the violence spread. Keeping this in mind, we will reconsider the system of the differential equations, (4.1) and (4.2), which originally gives us a general picture of the violence interaction. If we non-dimensionalized the system,

$\frac{d A}{d t}=\mu-\alpha A S$

$\frac{d S}{d t}=\alpha A S-\beta S$

With the characteristic scales $C_{A}=\frac{\beta}{\alpha}$ for the Abusive class $A, C_{S}=\frac{\beta}{\alpha}$ for the Susceptible and Domestic Violence Victim class $S$, and $C_{T}=\frac{1}{\beta}$ for time $t$, we end up with a nondimensionalized system of the differential equations as the following.

$\frac{d \tilde{A}}{d \tilde{t}}=\frac{\alpha \mu}{\beta^{2}}-\tilde{A} \tilde{S}$

$\frac{d \tilde{S}}{d \tilde{t}}=\tilde{S}(\tilde{A}-1)$ 
Where $\frac{\alpha \mu}{\beta^{2}}$ is a free parameter, $\rho$.

Now, we can easily notice that the free parameter, $\rho$, in the nondimensionalized system is same as the parametric conditions, (4.7) and (4.8). That means the overall behaviour of the violence can be observed by changing the free parameter, $\rho=\frac{\alpha \mu}{\beta^{2}}$ with the nondimensionalized equations. If we simply look at the nondimensionalized violence epidemic model, the free parameter, $\rho$, can be interpreted as recruitment to the population of a Violence-high-risk Tamale metropolitan, $N$; of course, the recruitment then immediately belongs to the Abusive class, $A$, as the violence spread is in progress. This phenomenon is similar to the fact that the original differential equations, (4.1) and (4.2), has the $\mu$ term, which represents new entrance to the Abusive class per unit time. Note that, in the dimensionless system of differential equations, the dimension of $\mu,[\mu]=\frac{P}{T}$ where $[A]=[S]=P$ and $[t]=T$

Recall the "forcing term" that we mentioned earlier. It becomes now clearer that the $\mu$ term acts like a "forcing term" which makes the behavior of the violence change. In fact, it is more accurate to say the free parameter, $\rho=\frac{\alpha \mu}{\beta^{2}}$, determines the behavior of the overall violence epidemic. The below figures visually present the different behavior of the violence spread at the equilibrium.

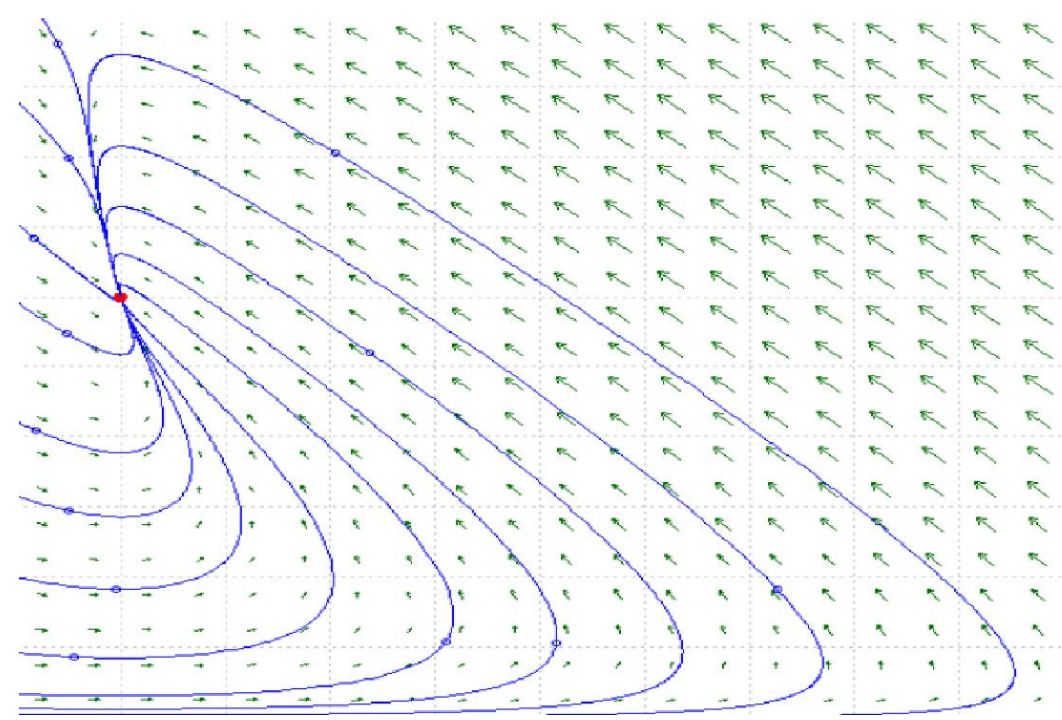

Fig.5: Graph showing phase plane when $\rho \geq 4$, the solutions in the phase-plane move to the Nodal sink equilibrium, $E\left(A^{*}, S^{*}\right)=\left(\frac{\beta}{\alpha}, \frac{\mu}{\beta}\right)$

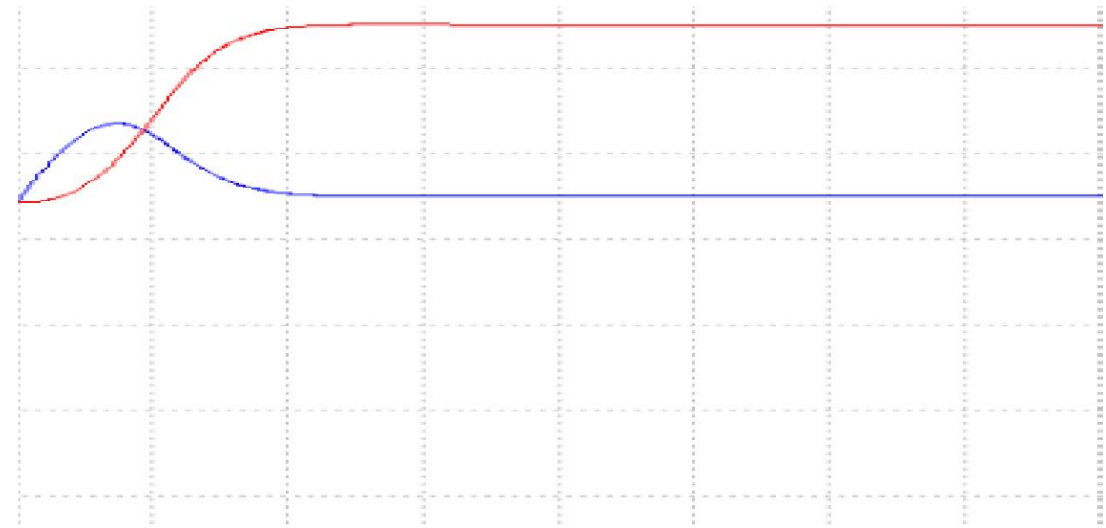

Fig. 6: Graph showing phase plane when $\rho \geq 4$, the curve below $(A)$ and the curve above $(S)$ approach to a steady state of the violence as $t \rightarrow \infty$. 


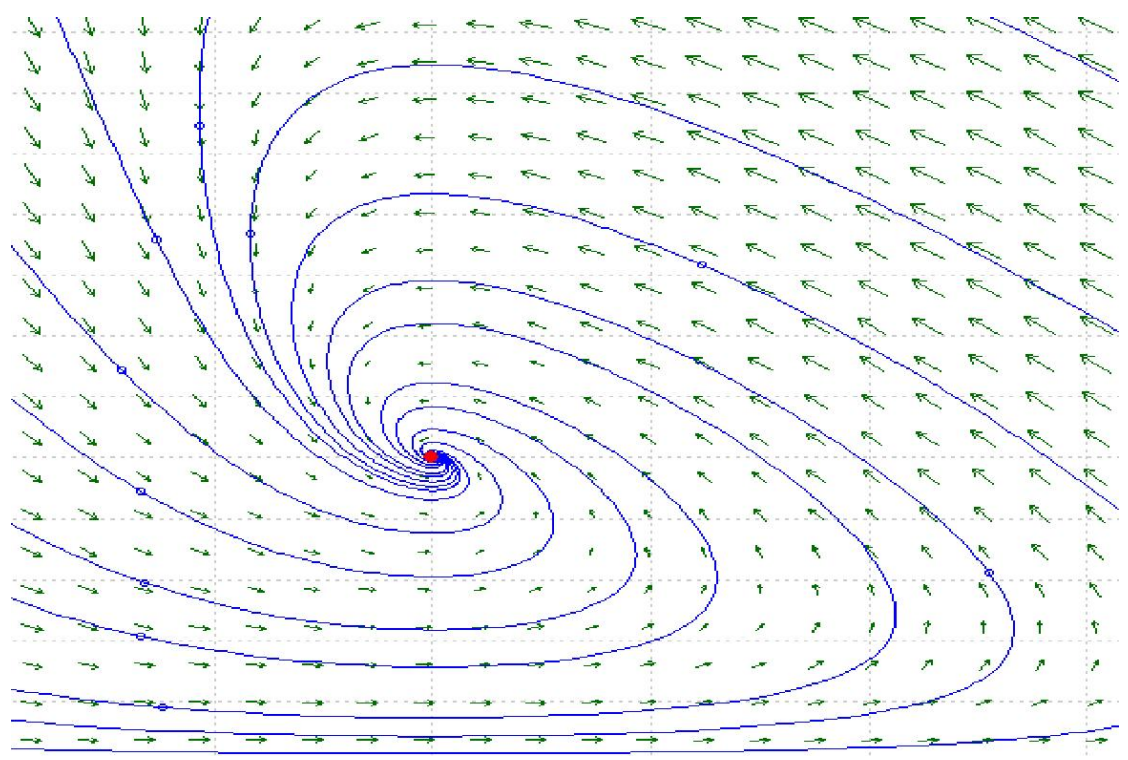

Fig. 7: Graph showing phase plane when $\rho<4$, the solutions in the phase-plane move to th spiral sink equilibrium, $E\left(A^{*}, S^{*}\right)=\left(\frac{\beta}{\alpha}, \frac{\mu}{\beta}\right)$.

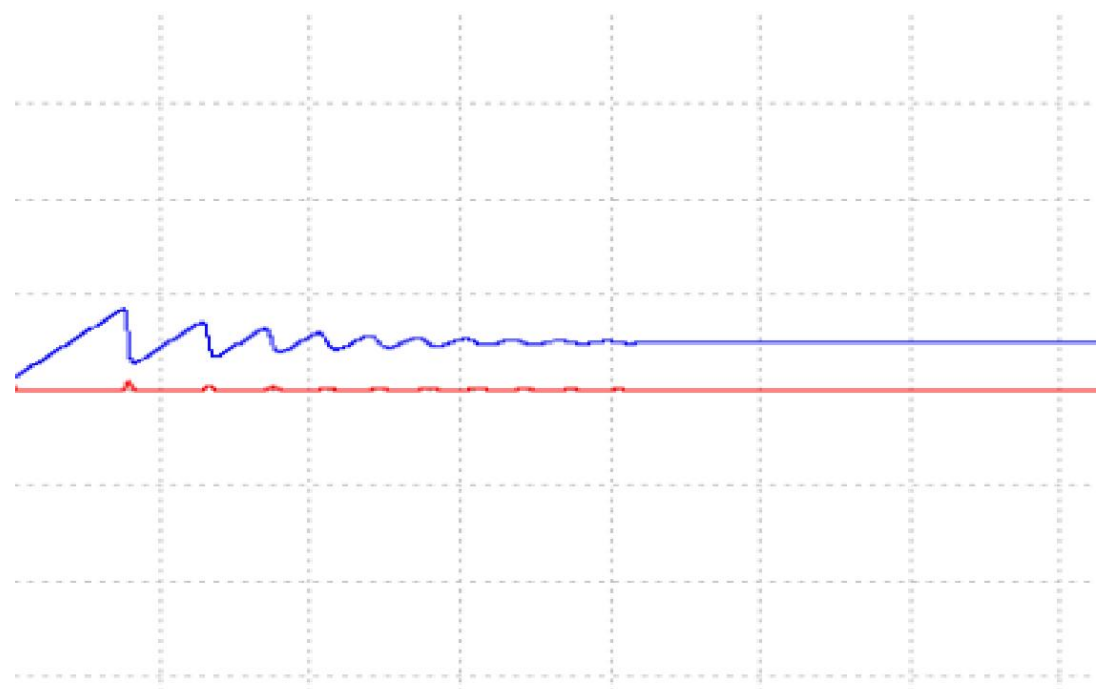

Fig. 8: Graph showing phase plane when $\rho<4$, the curve above $(A)$ and the curve below $(S)$ approach to a steady state, in oscillation manner, as $t \rightarrow \infty$

The general behavior of the violence epidemic as shown in the figures is predicted under the initial condition of the violence epidemic, starting close enough to the equilibrium point, and as time goes to infinity. Consider the differential equation (4.2) of the system, $\frac{d S}{d t}=\alpha A S-\beta S$.

At the very beginning of the violence epidemic, the populations of the susceptible individuals $(S)$ and the Domestic Violence Victims $(V)$ are almost negligible compared to the Abusive individuals $(A)$. Therefore, $N=A+S+V$, becomes $N \approx A$. Besides, the relatively long incubation time of Domestic Violence to the insignificance of the term $-\beta S$ which represent the conversion from the Susceptible to Domestic Violence Victim? Ignorance of Domestic Violence with most cases not been reported could also cause $-\beta S \approx 0$ during that particular time span. Now, according to these assumptions, we have an equivalent differential equation,

$\frac{d S}{d t}=\alpha N S$

By inspection, we can easily obtain the solution of the equation (4.9) is $S=S_{0} e^{\alpha N t}$. Clearly, $\alpha>0$ as long as the epidemic is in effect and $N>0$ since it's a population. Therefore, no matter how small the parameters are, the domestic Violence spread will exponentially grow in the beginning of the epidemic. This is what's actually happening in the real world at least in last 13 years according to the data from DOVVSU. 


\section{Summary of results}

The prevalent equilibrium $E\left(A^{*}, S^{*}\right)=\left(\frac{\beta}{\alpha}, \frac{\mu}{\beta}\right)$ is asymptotically stable. When $\rho \geq 4$, a relatively larger recruitment rate to the Abusive/Susceptible class, the violence epidemic slows down after some time. In this case, if a group of Susceptible/Unreported Victim is introduced to the Abusive/Susceptible class, the population of the Abusive/Susceptible class will approach to $A^{*}=\frac{\beta}{\alpha}$ while the populations of the Susceptible/Unreported Victim class approach to $S^{*}=\frac{\mu}{\beta}$, as time increases. On the other hand, when $\rho<4$, a relatively smaller recruitment rate to the Abusive/Susceptible class, the violence epidemic slow down with a certain period due to oscillation in terms of the model parameters while the population of $A$ and $S$ still approach to the same equilibrium point. However, in the very beginning of the violence epidemic, we could observe the violence spread obeying the Exponential Growth, $S=S_{0} e^{\alpha N t}$.

\section{Conclusion}

A simple continuous model can mirror a primary interaction of Domestic Violence spread, using a system of Ordinary Differential Equations called the Violence Epidemic Model (ASV). The modeling is accomplished by formulating the rate of change of each population with their interactions. According to the results, by changing an environmentalcontrol parameter, $\rho$, the long-term behavior of Domestic Violence against Women and children spread changes until the spread reaches a steady state. When $\rho \geq 4$, the solutions in the phase-plane move to the nodal sink equilibrium state

$E\left(A^{*}, S^{*}\right)=\left(\frac{\beta}{\alpha}, \frac{\mu}{\beta}\right)$. When $\rho<4$, the solutions in the phase-plane move to the spiral sink equilibrium state $E\left(A^{*}, S^{*}\right)=\left(\frac{\beta}{\alpha}, \frac{\mu}{\beta}\right)$. The result also shows that the Violence spreads in the very beginning obeying the Exponential growth model $S=S_{0} e^{\alpha N t}$

Knowing the simplified model might be somewhat inappropriate for the dynamics of Domestic Violence spread, though, fundamental mathematical modeling techniques are accomplished in this research to conceptualize a physical phenomenon.

\section{References}

[1] Gelles, R.J., \& Straus, M.A. (1979). "Determinants of violence in the family, toward a theoretical Integration”. New York: Free Press.

[2] Grais, RF; Ferrari, MJ; Dubray, C; Bjornstad, ON; Grenfell, BT; Djibo, A; Fermon, F; Guerin, PJ. (2006). estimating transmission intensity for a measles epidemic in Niamey, Niger: lessons for interven-tion: Transactions of the Royal Society of Tropical Medicine and Hygiene. 100: 867873.

[3] Hooten, MB; Anderson, J; Waller, LA. (2010) Assessing North American influenza dynamicswith a statistical SIRS model: Spatial and Spatiotemporal Epidemiology. 1: 177-185.

[4] Kuniya, T. (2011) Global stability analysis with a discretization approach for an age-structured multi-group SIR epidemic model: Nonlinear Analysis: Real World Applications. 12: 2640-2655.

[5] Li, X.Z, Li, W.S; Ghosh, M. (2009) Stability and bifurication of an SIR epidemic model with nonlinear incidence and treatment: Applied Mathematics and Computation. 210: 141-150.

[6] McGilchrist, C.A; McDonnell, L.F; Jorm, L.R; Patel, M.S. (1996) Log linear models using capture - recapture methods to estimate the size of a measles epidemic: Journal of Clinical Epidemiology. 49: 293-296.

[7] Montroll, E. W. (1978): Social dynamics and the quantifying of social forces. Proceedings of the National Academy of Sciences (USA), 75(10), 4633-4637.

[8] Munz, P; Hudea, I; Imad, J; Smith, RJ. (2009) Mathematical Modelling of an Outbreak of Zombie Infection: Infectious Disease Modelling Research Progress. Nova Science Publishers, Inc., pp. 133-150.

[9] Seager, J. (1997). "The state of women in the world: Atlas". London: Penguin Books Limited.

[10] Tuckwell, H.C, Williams, R.J (2007) some properties of a simple stochastic epidemic model of SIR type: Mathematical Biosciences. 208 : 76-97. 